\title{
Psoriatic Disease and Tuberculosis Nowadays
}

\author{
Nicola Balato, ${ }^{1}$ Luisa Di Costanzo, ${ }^{1}$ Fabio Ayala, ${ }^{1}$ Anna Balato, ${ }^{1}$ \\ Alessandro Sanduzzi, ${ }^{2}$ and Marialuisa Bocchino ${ }^{2}$ \\ ${ }^{1}$ Department of Dermatology, University of Naples Federico II, Via Pansini 5, 80131 Naples, Italy \\ ${ }^{2}$ Division of Respiratory Medicine, Department of Clinical and Experimental Medicine, University of Naples Federico II, \\ Monaldi Hospital, 80131 Naples, Italy
}

Correspondence should be addressed to Luisa Di Costanzo, luisadicostanzo@virgilio.it

Received 4 January 2012; Accepted 21 February 2012

Academic Editor: Luigina Romani

Copyright (c) 2012 Nicola Balato et al. This is an open access article distributed under the Creative Commons Attribution License, which permits unrestricted use, distribution, and reproduction in any medium, provided the original work is properly cited.

\begin{abstract}
Psoriasis is a chronic, relapsing and remitting inflammatory skin and joint disease that has a prevalence of 2-3\% in the world's population, whereas of 1-2\% in Europe. The traditional concept of psoriasis as the "healthy people's" disease has been recently revised because of ever-increasing reports of associations with various pathological conditions (hypertension, Crohn's disease, type II diabetes mellitus, obesity, dyslipidemia, metabolic syndrome, infectious conditions). Particularly, advances in psoriasis therapies have introduced biologic agents. All the tumor necrosis factor-alpha inhibitors are associated with an increased risk of developing active disease in patients with latent tuberculosis infection, because of TNF- $\alpha$ key role against Mycobacterium tuberculosis. For this reason, exclusion of active tuberculosis and treatment of latent tuberculosis infection are clinical imperatives prior to starting this therapy. Moreover active surveillance for a history of untreated or partially treated tuberculosis or latent form has already been shown to be effective in reducing the number of incident tuberculosis cases.
\end{abstract}

\section{Introuction}

Psoriasis is a chronic, relapsing and remitting inflammatory skin and joint disease that has a prevalence of $2-3 \%$ in the world's population [1], whereas of 1-2\% in Europe [2]. In Italy, the number of patients affected with psoriasis is estimated to be greater than 1.7 millions [3]. Men and women are equally affected and exhibit a bimodal distribution with a peak between 15 and 30 years of age and another between 50 and 60 years of age [1]. Psoriasis results from the interaction between genetic and environmental factors [4] and can cause significant impairment of physical, emotional, and psychosocial well-being of patients [5]. The traditional concept of psoriasis as the "healthy people's" disease has been recently revised [5] because of ever-increasing reports of associations with various pathological conditions, such as systemic diseases including hypertension, Crohn's disease, type II diabetes mellitus, obesity, dyslipidemia, metabolic syndrome, and infectious conditions [6]. Furthermore, psoriasis can also lead to substantial adverse socioeconomic consequences for patients, since physical disability and emotional distress of patients can affect their work functions.
For these reasons, psoriasis is associated with lesser work productivity and a greater number of missed work days compared to healthy individuals, incurring substantial indirect costs, and adding to the financial burden of the disease together with the incremental economic burden of treating comorbidities in addition to treating psoriasis compared to treating psoriasis alone $[7,8]$. Up to $30 \%$ of $70 \%$ of psoriatic patients require traditional systemic treatments, such as retinoids, methotrexate, and cyclosporine. Many of them imply long-term toxicity, treatment resistance, and potential drug interactions, so only $25 \%$ of psoriatic patients are completely satisfied with their treatment [9]. Advances in psoriasis therapies have introduced biologic agents, whose immune targeting is successful in treating many immunemediated inflammatory diseases. All the tumor necrosis factor-alpha (TNF- $\alpha$ ) inhibitors are associated with an increased risk of developing active disease in patients with latent tuberculosis infection (LTBI), because TNF- $\alpha$ is a key cytokine in protective host defense against Mycobacterium tuberculosis (Mtb) $[10,11]$. For this reason, exclusion of active tuberculosis (TB) and treatment of LTBI are, therefore, 
clinical imperatives prior to starting anti-TNF- $\alpha$ therapy and active surveillance for a history of untreated or partially treated TB or LTBI has already been shown to be effective in reducing the number of incident TB cases [12-14].

\section{Psoriatic Disease Pathogenesis}

The pathogenesis of psoriasis includes hyperproliferation as well as aberrant differentiation of keratinocytes, dermal angiogenesis, and inflammation. Dermal infiltration of inflammatory T cells, dendritic cells (DCs), macrophages, and neutrophils represents characteristic features of the disease [2]. Nowadays, the fundamental role played by the immune system in psoriatic disease pathogenesis is quite welldefined. T helper (Th)1 and Th17 lymphocytes contribute to the pathogenesis of psoriasis through the release of inflammatory cytokines that promote further recruitment of immune cells, keratinocyte proliferation, and sustained inflammation [15]. The T lymphocytes involved in lesion development were initially thought to be Th1 differentiated, based on interferon- (IFN-) gamma and interleukin- (IL-) 2 productions. Th17 cells have recently been classified as distinct from Th1 and Th2 subsets. They are defined by the ability to synthesize IL-17 in response to antigen-presenting cell-derived IL-23 and other differentiating cytokines. In addition, Th17 cells have been reported to cosynthesize IL17 and IFN-gamma as well as IL-22 [16]. Recently, psoriatic skin lesions are reported to have increased gene expression of IL-23, IL-17, and IL-22 [17]. IL-23 is a heterodimeric cytokine composed of two subunits (p40 subunit, common with IL-12, and p19 subunit, specific for IL-23) [18]. IL23 is produced by dendritic cells (DCs), macrophages, and other antigen-presenting cells under the influence of some Gram-positive as well as Gram-negative bacteria and lipopolysaccharides [18]. Several recent studies suggest that psoriasis is a Th17 cell-mediated disease driven by IL-23 [19]. Moreover, TNF- $\alpha$ stimulates CD $11^{+}$inflammatory DCs to produce IL-23 and IL-20 and appears to be a critical cytokine for many of the clinical features of psoriasis, including keratinocyte hyperproliferation, endothelial cell regulation, and recruitment/effector function of memory $\mathrm{T}$ cells. All these findings reinforce that psoriasis pathogenesis is a complex interaction among genetic, immunological, and environmental components.

\section{Clinical Phenotype and Histological Psoriasis Features}

Clinical diagnosis of psoriasis is relatively easy for a dermatologist, especially when the lesions present as erythematous, sharply demarcated indurated plaques with silvery white scales. Plaques may have an oval or irregular shape, varying from one to several centimetres in diameter and are usually distributed symmetrically on the extensor surfaces of limbs (mainly elbows and knees), the lower back and the scalp. Itching is variable, but it is usually absent [20]. These clinical aspects reflect the histopathological findings observed in active lesions, characterized by hyperkeratosis, parakeratosis, diminution, or loss of the granular cell layer, acanthosis of the epidermal ridges, tortuous and dilated blood vessels, and perivascular leukocytic infiltrate in the dermal papillae [1]. The clinical and histological features of chronic plaque psoriasis are generally sufficient to make the diagnosis. Furthermore, psoriasis can present many faces, including guttata, pustular, and erythrodermic. Guttate psoriasis is characterized by the acute onset of round, erythematous, slightly scaling papules over the trunk and extremities. The face could be involved. The disease is self-limiting; however, a proportion of affected individuals may progress to a more chronic form of plaque psoriasis. Flares of guttate lesions may appear during the course of chronic plaque psoriasis and can follow streptococcal infection (particularly of the upper respiratory tract) and/or acute stressful life events [21]. Generalized pustular psoriasis, as well as the localized form and its variants (circinate or Bloch-Lapière's pattern, acrodermatitis continua of Hallopeau) are characterized by nonfollicular sterile pustules, which represent the macroscopic aspect of the massive neutrophil infiltration of epidermis $[21,22]$. The erythrodermic form is dominated by generalized erythema, loss of peculiar clinical features of psoriasis, and skin failure, that is, inability to maintain homeostatic functions [23]. Psoriatic erythroderma is not substantially different from erythroderma by other causes.

\section{Psoriasis and Metabolic Comorbidities}

It has recently been found that psoriatic patients have a higher prevalence of some metabolic disorders [24], particularly obesity, diabetes, or abnormal glucose intolerance, dyslipidemia, and systemic hypertension, which together are known as the metabolic syndrome [25]. Psoriasis is now also considered to be a marker of increased cardiovascular risk, especially in young patients [26]. Psoriatic disease is associated with unhealthy behaviors, particularly smoking and obesity; in addition, it may influence metabolic and cardiovascular risk independently of lifestyle factors, through common genetic risks, resulting in a chronic systemic inflammatory pathway [27]. A recent study, evaluating the association with comorbidities in psoriasis patients in Italy, showed that, from a total sample of 511, 532 individuals, overall patients had more selected comorbidities compared to healthy controls, in particular chronic ischemic heart disease, obesity, diabetes mellitus, bronchitis, cardiac valve abnormalities, dermatomycosis, benign mammary dysplasias, disorders of penis, disorders of external ear, inflammation of eyelids, and contact dermatitis. In agreement with previous studies, they found a significant association of psoriasis with cardiovascular risk factors (diabetes mellitus and obesity) without, however, confirming an association with others (dyslipidemia and blood hypertension). In contrast, we found no significant difference in general medical history (e.g., cardiac diseases, diabetes) between psoriatic and control groups, except for high blood pressure that was more prevalent in psoriatic patients [28-31].

A causal link between psoriasis and cardiovascular disease is hypothesized also for the involvement of the same 
mediators and markers of inflammation, mainly TNF- $\alpha$, IL-6, fibrinogen, and C-reactive protein [6]. Apart from these cytokines, insulin-like growth factor (IGF)-I, the main anabolic mediator of somatotroph axis also acting as an autocrine/paracrine signal essential for proliferation of epidermal keratinocytes, has been found to be overproduced in psoriatic epidermis. Despite the increase in IGF-I in psoriatic plaques, psoriatic patients exhibited low circulating levels of IGF-I, with a negative correlation to Psoriasis Area and Severity Index (PASI). However, it is well known that a number of inflammatory cytokines affecting IGFI secretion and subtle changes in IGF-I levels have been associated with unfavourable lipid profiles, with increased cardiovascular mortality [32]. Thus, although abnormalities in somatotroph axis activity have been hypothesized to account for the low IGF-I levels in the psoriatic patients, with a possible primary or secondary effect of these disturbances on the psoriasis process modulation [33], the more likely association of low IGF-I with the common inflammatory pathways of both metabolic syndrome or psoriasis has not been considered as far. On this basis, Savastano et al. speculated that in psoriasis chronic inflammation might be an important modulator of low IGF-I status and that, similarly to other pathological conditions, low IGF-I status could be added as a further possible mechanistic link between psoriasis and associated metabolic comorbidities [34]. In conclusion, psoriatic systemic inflammation may underneath insulin resistance, which in turn triggers endothelial cell dysfunction, leading to atherosclerosis and finally myocardial infarction or stroke [27].

\section{Psoriasis and Malignancy}

Although anti-TNF- $\alpha$ drugs mechanism of action has been well investigated, long-term studies concerning malignancy risk associated with these immunosuppressive agents have been most extensively performed in rheumatoid arthritis more than in psoriasis population; there are in fact just some case reports regarding this matter in psoriasis, suggesting that these therapies can permit malignant processes [35]. Therefore, risk of malignancy with anti-TNF- $\alpha$ in psoriasis remains unclear. However, the majority of reports indicate that TNF$\alpha$ inhibitors may cause a slightly increased risk of cancer, including nonmelanoma skin cancer and hematologic malignancies [36, 37]. So far, it is worthy that oncologic personal and familiar history, skin examination, and baseline blood tests attempting to identify any hematologic abnormalities be required before starting biologics therapy [35].

\section{Psoriatic Infectious Co morbidities (Other than TB)}

TNF- $\alpha$ plays an important role in host defense and anti-TNF$\alpha$ agents may theoretically increase the risk of infections. Most recent studies suggest that anti-TNF- $\alpha$ agents are associated with a slight increased risk of serious infections, especially in the early phase of treatment and an absolute rate of infections relatively low [38]. Grijalva analyzed whether initiation of TNF- $\alpha$ antagonists compared with nonbiologic drugs was associated with an increased risk of serious infections in a cohort of patients affected by rheumatoid arthritis, inflammatory bowel disease, psoriasis, psoriatic arthritis, or ankylosing spondylitis; rates were 5.41 for TNF$\alpha$ antagonists and 5.37 for traditional systemic drugs per 100 person-year, showing no significant difference between the 2 groups [39]. Another recent systematic review showed that there may be a small increased risk of overall infection with short-term use of TNF- $\alpha$ antagonists in psoriasis, whereas $97.6 \%$ were nonserious infections and the large majority of these were ones of the upper respiratory tract [40].

\section{Psoriasis and TB}

Recently, infliximab, etanercept, adalimumab, and golimumab have become the drugs of choice in the treatment of these disorders. Of course, such kinds of drugs could conceptually interfere with a cytokine, TNF- $\alpha$, which is crucial in the development and maintenance of the granuloma. Moreover, the early diagnosis and treatment of individuals harboring the $\mathrm{Mtb}$ is key to ensuring the effectiveness of health programs aimed at the elimination of TB. On the other hand, psoriasis "per se" could represent an independent risk factor for TB since, interestingly, an unexpected high prevalence was found in patients affected by such a disease (18.0\%), even adjusting for age, work, and other parameters [41]. A similar result is reported by Bassukas et al. during a two-year period, LTBI diagnosis rate was compared in consecutive patients with psoriasis or inflammatory bowel disease like Crohn's disease or ulcerative colitis: these patients had significantly smaller tuberculin skin testing compared to psoriasis patients $(P=0.007)$. Applying LTBI diagnosis guidelines, latent infection resulted in more psoriasis $(50 \%)$ than inflammatory bowel disease patients $(24.2 \%)$, prior to onset of any anti-TNF- $\alpha$ treatment $(P=0.04)$ [42]. A recent survey concerning the evaluation of the infectious complications during biological therapy of psoriasis showed a rate of infections of $12.24 \%$, with only one case of pulmonary TB, out of 988 patients [43]. The authors stressed that such a result depend on a strict screening of LTBI, prior to starting the biological treatment. A French report showed that, in a mixed population of patients treated with TNF- $\alpha$ blockers, including psoriasis, 45 cases were collected of non-TB opportunistic infections (OIs). One-third (33\%) of OIs were bacterial (4 listeriosis, 4 nocardiosis, 4 atypical mycobacteriosis, 3 nontyphoid salmonellosis), $40 \%$ were viral (8 severe herpes zoster, 3 varicella, 3 extensive herpes simplex, 4 disseminated cytomegalovirus infections), $22 \%$ were fungal (5 pneumocystosis, 3 invasive aspergillosis, 2 cryptococcosis), and $4 \%$ were parasitic (2 leishmaniasis). Ten patients (23\%) required admission to the intensive care unit, and four patients $(9 \%)$ died. Risk factors for OIs were treatment with infliximab $(\mathrm{OR}=17.6(95 \%$ CI 4.3-72.9); $P<0.0001$ ) or adalimumab ( $\mathrm{OR}=10.0$ (2.3 to 44.4$) ; P=$ 0.002 ) versus etanercept, and oral steroid use $>10 \mathrm{mg} /$ day or intravenous boluses during the previous year $(\mathrm{OR}=6.3$ (2.0 to 20.0); $P=0.002$ ) [44]. Another study identified 
69 cases of tubercular active disease prospectively through the French RATIO registry: the sex and age-adjusted TB incidence rate was 1.17 per 1,000 patient-years, 12.2 times that of the general population [45]. A similar conclusion was reached by a Portuguese biologics registry study that found the TB risk with anti-TNF- $\alpha$ antibodies to be 12fold greater than with etanercept [46]. Sánchez-Moya et al. report that, among one hundred and forty-four patients with moderate-to-severe psoriasis treated with anti-TNF- $\alpha$ agents, a total of $42(29 \%)$ patients were diagnosed with LTBI based on a positive tuberculin skin test (TST) or reTST, and/or signs of past TB in the chest X-ray. All of them received chemoprophylaxis with isoniazid $(\mathrm{H})$. Only one patient developed an active lymphnode TB [47]. Besides, the risk of active $\mathrm{TB}$ due to infliximab appeared to be twice that of etanercept [48]. Most of the active TB cases in patients treated with TNF antagonists are due to reactivation of latent infection with Mtb. TB in patients who have been treated with TNF antagonist therapies usually progresses rapidly and is frequently disseminated, with several extra-pulmonary localizations. Thus, the most effective way to avoid TB reactivation is (and remains) treatment of the latent infection.

\section{Management of TB Infection in Psoriasis Patients}

8.1. Conventional TST and Interferon Gamma Release Assays: Overview on Test Characteristics. Screening for LTBI before the initiation of any immune-suppressive therapy regimens, including TNF- $\alpha$ blocking agents, is part of current management strategies of common inflammatory disorders such as psoriasis, such an approach having been associated with an estimated reduction of more than $80 \%$ of the risk of TB reactivation $[49,50]$. Guidelines for TB prevention suggest taking a careful medical history and excluding active TB before starting any kind of treatment. All patients should be questioned regarding their demographic details (i.e., age and country of birth), history of previous Bacillus CalmetteGuèrin (BCG) vaccination, TB risk factors (i.e., recent close exposure to active TB cases, immigration from or recent stay in high TB prevalence areas, and chest X-ray evidence of TB sequelae), and current treatments (i.e., drugs) [51].

Diagnostic algorithms based on the use of the TST are still in use worldwide for the detection of TB infection. As known, TST is a measure of a delayed-type-hypersensitivity response to the intradermal inoculation of PPD (purified protein derivative), which is a mixture of more than 200 mycobacterial antigens. Despite TST is easy, safe, and inexpensive to administer, poor specificity limits its use because of PPD cross-reactivity with environmental mycobacteria and with the $M$. bovis BCG vaccine strains. In addition, despite different cut-off values are used to stratify PPD reactors according to their likelihood to be infected and to predict the risk of disease progression, sensitivity of TST is significantly reduced in the case of immune-compromise where a TST-positivity is given by an induration area equal or greater than $5 \mathrm{~mm}[51,52]$. Finally, TST result may not be stable over time as the reaction size may increase due to a new infection (conversion) or to serial testing in previously sensitized individuals (boosting). Reversion to a negative TST result may also occur [53]. Overall, these limitations explain why in the guidelines released by the British Thoracic Society (BTS) for the management of patients due to start anti-TNF therapies, the use of TST was not recommended in patients with no TB risk factors, while remaining controversial in high-risk cases due to the expected high rate of false-negative results [54].

Recently, commercially available and FDA (Food and Drug Administration) approved interferon gamma release assays (IGRAs) have been introduced in clinical practice as alternative tools for the identification of Mtb infection. Unlike TST, blood tests are based on the principle of detecting IFN- $\gamma \square$ production by effector memory T-cells upon short term (16-20 hrs) in vitro stimulation with TBspecific antigens. Measurements of IFN- $\gamma$ are performed either by ELISpot-based assay (T-SPOT.TB, TS-TB, provided by Immunotech, UK) or ELISA [QuantiFERON TB Gold (QFT-G) and QFT-in-tube (QFT-IT), both provided by Cellestis, Australia]. All tests rely on the use of two TB specific antigens, that are early secretory antigen- (ESAT-) 6 and culture filtrate protein- (CFP-) 10, mapped to a genomic region called $\mathrm{RD}$ (region of difference)-1, which is absent in the vaccine strains and in most non-TB mycobateria (expect kansasii, szulgai, marinum, flavescens, and gastrii) [55]. To date, QFT-IT is the latest improvement of the ELISA technology. It has widely replaced the previous inplate format (QFT-G) as blood samples are directly collected into tubes pre-coated with antigens (also including a third antigen, that is, the RD-11-related TB7.7) and ready for incubation. Each test is provided of both a negative and a positive control (phytohemagglutinin, PHA), thus allowing a more comprehensive evaluation of the host immune reactivity. Despite there is evidence that IGRAs performance (i.e., QFT) may significantly vary in comparison to TST depending on the epidemiological and clinical setting [56, 57], overall, a pooled sensitivity of $87.5 \%$ and $81 \%$ has been estimated in a recent meta-analysis, respectively, for TS-TB and QFT-IT, as compared to TST (70\%). Pooled specificity has instead been evaluated at $86 \%$ for TS-TB and $99 \%$ for QFT-IT. The pooled estimated rate of indeterminate results was low, 2.1\% (95\% CI, 0.02-0.023) for QFT-IT and $3.8 \%(95 \% \mathrm{CI}, 0.035-0.042)$ for TS-TB, increasing to $4.4 \%$ (95\% CI, 0.039-0.05) and 6.1\% (95\% CI, 0.052-0.071), respectively, among immunecompromised hosts [58]. As for TST, a main limitation of IGRAs is represented by the lack of discrimination of LTBI from active TB. Reproducibility in serial testing along with definitions of conversion and reversion, differentiation of cut-off values for targeting selected patient populations, positive predictive value, and boosting remain as further areas of uncertainty that only in part have recently been addressed, as elsewhere discussed [59, $60]$. In conclusion, IGRAs testing requires the availability of equipped laboratories with expertise in the field and adequate economic resources to ensure efficient samples turnover. Although intrinsic technical characteristics may explain a certain degree of discrepancy when comparing the performance of the two IGRA formats, current clinical 
evidence actually does not clearly favor one test over the other in any setting.

8.2. TST and Advances on IGRAs Performance in Psoriasis Patients. Nonetheless, some recent evidence still supports that the use of TST is reliable as an effective diagnostic approach for the detection of TB infection, as suggested by Sanchez-Moya et al. in a prospective evaluation of 144 patients affected by moderate-to-severe psoriasis in Spain [47], some concerns on TST application in this selected patient population need to be addressed. TST may be ineffective due to the dubious results that it generates in patients with psoriasis as disease activity may substantially affect the test outcome [60]. First, it may be impossible to find lesion-free skin areas suitable for TST in patients with severe skin disease. Secondly, the observation of an increased TST reactivity of even healthy skin regions has been associated with proinflammatory priming that leads to an over-reaction to a wide spectrum of antigenic triggers [61]. In this issue, Bassukas et al. [42] have recently shown that patients with moderate-to-severe plaque psoriasis had significantly larger TST reactions compared to patients with inflammatory bowel disease, as previously reported. To overcome TST limitations, assessment of the value of IGRAs as diagnostic tools in detecting LTBI in psoriasis patients is currently under investigation, most of the studies being focused on patient candidates for receiving anti-TNF agents due to the high impact of such a therapy on TB risk [62, 63]. Chiang et al. [64] in a recent prospective study first used TS-TB as a unique diagnostic tool for TB infection screening purposes in 63 patients affected by severe psoriasis in UK. The Authors found a prevalence of LTBI of 7.9\%, the IGRA test positivity being associated with a travel history to $\mathrm{TB}$ endemic countries. A retrospective analysis carried out in Switzerland over a 4 -year period on 50 psoriasis patients, the $90 \%$ with prior BCG vaccination, subjected to both TST and TS-TB before the initiation of anti-TNF treatment showed that a positive TS-TB result was strongly associated with a presumptive diagnosis of LTBI, while this was not the case for TST. Agreement between the two tests was quite poor $(\kappa=0.33)$. LTBI treatment was avoided in the $20 \%$ of patients tested positive by TST $(\geq 5 \mathrm{~mm})$ but negative by TS-TB (however, one case of disseminated tuberculosis occurred despite LTBI treatment after 28 weeks of therapy with adalimumab) [64]. More recently, a further retrospective study conducted in Taiwan, an intermediate TB burden country, has assessed the accuracy of QFT in 147 psoriasis patients, including both cases with and without treatment with TNF-alpha blocking agents (median exposure of 24 weeks) [65]. Previous survey reports in this setting have reported a TST-positive rate of $47 \%$ in the general population aged 20-59 years old [66]. In the study by Chiu et al. the percentages of LTBI in psoriasis patients ranged from $30 \%$ to $36 \%$, according to TST cut-off used, that was 10 or $5 \mathrm{~mm}$, respectively, the overall rate of QFT positive tests being even lower (12\%). Agreement with TST was poor $(63 \%, \kappa=0.046)$. Despite this, unnecessary treatment of LTBI was avoided in a significant number of cases, active TB developed in one out of four QFT-positive patients (25\%) that while receiving TNF blocking agents were not treated for LTBI [65]. Finally, a cross-sectional study realized in Brazil has shown that the frequency of TST-positive responses and skin induration size were significantly lower in 33 psoriasis patients $(18 \% ; 2.6 \pm 0.7 \mathrm{~mm})$ as compared to 30 cases affected by other common dermatological diseases (control group) (53\%; $9.3 \pm 1.4 \mathrm{~mm})$ [67]. Conversely, frequencies of TSTB-positive results were not different in psoriasis (47\%) and control patients $(40 \%)$, while a poor agreement with TST was recorded in the formers $(\kappa=0.375)$. These findings confirm a previous observation of a decrease of central memory anti TB immune responses in untreated psoriasis patients living in endemic areas while they retained T-cell memory effector activity [68]. Overall, rates of indeterminate results were quite low ranging from 1.6\% (TS-TB) [63] to 1.9\% (QFT-IT) [65]. As indeterminate results may reflect a high background IFN-gamma production (negative control) or, alternatively, the inability of the immune system to mount a T-cell response (positive control), every attempt should be made to clarify the reasons, also excluding technical errors, behind this kind of results [69]. Head-to-head comparison studies of TS-TB versus QFT are even more limited. In this issue, we simultaneously tested a small cohort of patients affected by psoriasis or psoriatic arthritis with TST in comparison with both TS-TB and QFT-IT. The main finding of the study was a good agreement of blood tests with conventional TST $(\kappa=0.86$ and 0.84 , resp.). This was not surprising due to the high rate of negative results recorded as patients were mainly represented by non-BCG vaccinated young individuals without known TB risk factors, confirming a previous report [70]. However, in two TST-negative cases (11\%), IGRAs yielded a positive result that allowed the identification of a presumptive LTBI [71], thus supporting using these assays in psoriasis patients [72]. Finally, only a few studies to date report on the performance of TST or IGRAs for monitoring patients already under treatment with TNF- $\alpha$ blockers. Single-case reports and case series have described psoriasis patients tested negative by TST but positive by means of an IGRA [73, 74]. Clinical applicability of QFT-IT has been prospectively assessed in 50 patients with psoriasis along with TST while patients were on anti-TNF therapy. Agreement among tests was moderate $(\kappa=0.408)$ at baseline, good $(\kappa=0.734)$ at 6 months, and fair $(\kappa=$ 0.328 ) at 12 months of treatment [75]. To date, TST+/QFTcases were regarded as not suffering from LTBI which instead was 12 diagnosed based on QFT conversion alone. Overall, current evidence including disease conditions other than psoriasis suggests IGRAs results to be not interpretable in this setting [76-78]. Longitudinal high-powered studies with longer followup periods are necessary to optimize their use and systematically assess whether IGRAs can be used in this clinical scenario for monitoring a previous infection or for regular screening purposes in high prevalence countries or more specifically after an exposure event in low burden areas in the case of occurrence of a new infection.

8.3. National Guidelines and Consensus Recommendations: Points of Agreement and Criticisms. Actually, views on how TST and IGRAs should be employed for LTBI screening 
are widely divergent and no specific recommendations in the setting of patient candidates for receiving immunesuppressive therapies are available [79]. Among the disease conditions for which LTBI diagnosis is mandatory, psoriasis occupies a unique position due to some specific diseaseassociated issues. Overall, the present observations suggest that TST-based screening algorithms may lead to overdiagnosis of LTBI in patients affected by overt plaque psoriasis or, conversely, may be not applicable in the case of extensive skin involvement. In addition, a likely impairment of central memory T-cell responses leading to TST unresponsiveness may further represent an issue of concern in these patients, at least in TB endemic areas. This means that innovative approaches are needed to overcome these limitations through the readaptation of current guidelines while suggesting a key role of IGRAs as first-line diagnostics for putative LTBI at least in patients with diffuse skin morbidity and in the case of confounding factors, like previous BCG vaccination. In 2005, the U.S. Centers for Disease Control and Prevention (CDC) first recommended the use of QFT-G (the plate ELISA-based format available at that time) instead of TST in all circumstances and clinical settings in which TST was used [80]. However, this is not the same approach recommended in the last update where different strategies are suggested including testing with both an IGRA (with no test format preference) and a TST in the case of high suspicion of false-negative or false-positive results, indeterminate, borderline, or invalid results [81]. In the European setting in 2006, the National Institute for Clinical Excellence (NICE) guidelines proposed a cost-effective two-step strategy that is confirmation of a positive TST result by any available IGRA [82]. It was, however, suggested to consider IGRAs as an alternative tool in the case of not reliability of TST or of high suspicion of false-negative TST results in immunecompromised patients. Later on, IGRA tests were offered as a suitable alternative to TST in all BCG-vaccinated individuals within the context of this risk assessment by the Heath Protection Agency (HPA) [83]. Despite the NICE guidelines were not focused on the management of patients to be treated with TNF blockers, Lalvani has recently suggested that it may be prudent to perform both TST and any IGRA to maximise diagnostic accuracy until stronger evidence on blood tests use in this patient population has expanded sufficiently [55]. Despite the Canadian and Italian guidelines which are in line with the NICE approach $[84,85]$, further recommendations by national societies worldwide still have divergent positions when targeting patient candidates to immune-suppressive/anti-TNF therapies. To date, most of them, except those from Switzerland and Germany, recommend TST (mainly as a one-step strategy) as the unique screening tool, with cut-off values ranging from 5 to $10 \mathrm{~mm}$ in the different geographic settings. Conversely, any commercial IGRA is preferred instead of TST in Switzerland, while Germany guidelines recommend the combined use of TST (cut off $\geq 5 \mathrm{~mm}$ ) only in IGRA-negative cases with clinically proven TB exposure $[45,50,86-93]$. The more recent TBNET consensus statement [48], based on published evidence and expert opinions, suggests using any IGRA, or, as an alternative, the TST testing one-step strategy (cut off $\geq 10 \mathrm{~mm}$ ) in patients with no prior BCG vaccination. Repeated testing for $\mathrm{TB}$ infection may be considered in patients with ongoing risks of exposure. In this case, the use of TST is not strictly recommended due to the boosting effect. Overall, in all the scenarios proposed, no differences among disease conditions to be targeted are considered and no specific recommendations are proposed to tailor specific disease phenotypes, such as severe skin psoriasis. In this issue, the unique document specifically focused on psoriasis patients provided by the National Psoriasis Foundation suggests the use of TST as first-line screening tool (cut-off equal or greater than $5 \mathrm{~mm}$ ) and considering IGRA testing in BCG-vaccinated patients. However, there is no mention of specific disease subtypes [87].

8.4. Treatments Options for LTBI and Active TB. As recently summarized in the TBNET consensus statement, recommended treatment regimens for LTBI vary, their efficacy having not been evaluated in this setting. They mainly include 6 or 9 months with $\mathrm{H}, 3$ months of rifampicin (R) plus $\mathrm{H}$, and 4 months of $\mathrm{R}$ [48]. The time delay before starting anti-TNF agents also differs ranging from 3 weeks to as long as possible after the initiation of TB prophylaxis, depending on the geographical context $[45,50,86-93]$. Strict adherence to treatment has to be strongly encouraged as it significantly reduces the patient risk to develop active TB. Patients should be educated about signs and symptoms of possible TB reactivation or of drug-induced side effects. Baseline and routine followup of liver enzymes should be performed on a monthly basis. Repeated chest X-ray is not recommended. Imaging of the thorax also including highresolution-computed tomography scan should instead be performed without delay in the case of suspected active TB. No action is required for patients having completed a previous course of anti-TB treatment unless a reinfection is plausible. Treatment regimens are recommended in the case of active TB according to international standards as for susceptible immune-competent patients [94]. No differences in treatment duration have been suggested as actually there is no evidence for the need of any prolongation. The optimal timing for the initiation of TNF-blocking therapies is unclear, in some instances being recommended after the completion of at least 2 months of anti-TB treatment. Expert opinion in agreement with the CDC conversely suggests waiting until the end of a full course. Maintaining vigilance for $\mathrm{TB}$ even after the completion of appropriate treatment strategies (LTBI/active $\mathrm{TB}$ ) remains of utmost clinical relevance.

\section{Abbreviations}

$\begin{array}{ll}\text { TNF- } \alpha: & \text { Tumor Necrosis Factor- } \alpha \\ \text { TB: } & \text { Tuberculosis } \\ \text { Mtb: } & \text { Mycobacterium tuberculosis } \\ \text { LTBI: } & \text { Latent tuberculosis infection } \\ \text { DCs: } & \text { Dendritic cells } \\ \text { Th: } & \text { T helper } \\ \text { IFN-gamma: } & \text { Interferon-gamma } \\ \text { IGF-I: } & \text { Insulin-like growth factor-I }\end{array}$




$\begin{array}{ll}\text { PASI: } & \text { Psoriasis Area and Severity Index } \\ \text { OIs: } & \text { Opportunistic infections } \\ \text { TST: } & \text { Tuberculin skin test } \\ \text { H: } & \text { Isoniazid } \\ \text { R: } & \text { Rifampicin } \\ \text { BCG } & \text { Bacillus Calmette-Guèrin } \\ \text { PPD: } & \text { Purified protein derivative } \\ \text { BTS: } & \text { British Thoracic Society } \\ \text { FDA: } & \text { Food and Drug Administration } \\ \text { IGRAs: } & \text { Interferon gamma release assays } \\ \text { T-SPOT.TB, TS-TB: } & \text { ELISpot-based assay } \\ \text { QFT-G: } & \text { ELISA QuantiFERON TB Gold } \\ \text { QFT-IT: } & \text { QFT-in-tube } \\ \text { ESAT-6: } & \text { Early secretory antigen-6 } \\ \text { CFP-10: } & \text { Culture filtrate protein-10 } \\ \text { RD-1: } & \text { Region of difference-1 } \\ \text { PHA: } & \text { Phytohemagglutinin } \\ \text { CDC: } & \text { Disease control and prevention } \\ \text { NICE: } & \text { National Institute for Clinical } \\ & \text { Excellence } \\ \text { HPA: } & \text { Heath Protection Agency. }\end{array}$

\section{References}

[1] G. K. Perera, P. Di Meglio, and F. O. Nestle, "Psoriasis," Annual Review of Pathology, vol. 7, pp. 385-422, 2012.

[2] C. M. Sweeney, A.-M. Tobin, and B. Kirby, "Innate immunity in the pathogenesis of psoriasis," Archives of Dermatological Research, vol. 303, no. 10, pp. 691-705, 2011.

[3] R. Saraceno, R. Mannheimer, and S. Chimenti, "Regional distribution of psoriasis in Italy," Journal of the European Academy of Dermatology and Venereology, vol. 22, no. 3, pp. 324-329, 2008.

[4] E. Christophers, "Psoriasis-epidemiology and clinical spectrum," Clinical and Experimental Dermatology, vol. 26, no. 4, pp. 314-320, 2001.

[5] G. A. Vena, G. Altomare, F. Ayala et al., "Incidence of psoriasis and association with comorbidities in Italy: a 5-year observational study from a national primary care database," European Journal of Dermatology, vol. 20, no. 5, pp. 593-598, 2010.

[6] B. B. Davidovici, N. Sattar, P. C. Jörg et al., "Psoriasis and systemic inflammatory diseases: potential mechanistic links between skin disease and co-morbid conditions," Journal of Investigative Dermatology, vol. 130, no. 7, pp. 1785-1796, 2010.

[7] A. B. Kimball, A. Guérin, M. Tsaneva et al., "Economic burden of comorbidities in patients with psoriasis is substantial," Journal of the European Academy of Dermatology and Venereology, vol. 25, no. 2, pp. 157-163, 2011.

[8] A. P. Yu, J. Tang, J. Xie et al., "Economic burden of psoriasis compared to the general population and stratified by disease severity," Current Medical Research and Opinion, vol. 25, no. 10, pp. 2429-2438, 2009.

[9] R. S. Stern, T. Nijsten, S. R. Feldman, D. J. Margolis, and T. Rolstad, "Psoriasis is common, carries a substantial burden even when not extensive, and is associated with widespread treatment dissatisfaction," Journal of Investigative Dermatology Symposium Proceedings, vol. 9, no. 2, pp. 136-139, 2004.

[10] M. Lebwohl, J. Bagel, J. M. Gelfand et al., "From the Medical Board of the National Psoriasis Foundation: monitoring and vaccinations in patients treated with biologics for psoriasis,"
Journal of the American Academy of Dermatology, vol. 59, no. 2, pp. 209-217, 2008.

[11] A. B. Kimball, D. Gladman, J. M. Gelfand et al., "National Psoriasis Foundation clinical consensus on psoriasis comorbidities and recommendations for screening," Journal of the American Academy of Dermatology, vol. 58, no. 6, pp. 10311042, 2008.

[12] A. Lalvani and K. A. Millington, "Screening for tuberculosis infection prior to initiation of anti-TNF therapy," Autoimmunity Reviews, vol. 8, no. 2, pp. 147-152, 2008.

[13] H. Haake, J. Köneke, K. Amann, J. Vom Dahl, and U. Janssen, "Development of systemic lupus erythematosus with focal proliferative lupus nephritis during anti-TNF- $\alpha$ therapy for psoriatic arthritis," Medizinische Klinik, vol. 102, no. 10, pp. 852-857, 2007.

[14] B. P. Vaughn, G. A. Doherty, S. Gautam et al., "Screening for tuberculosis and hepatitis B prior to the initiation of anti-tumor necrosis therapy," Inflammatory Bowel Diseases. In press.

[15] Y. Chung, S. H. Chang, G. J. Martinez et al., "Critical Regulation of Early Th17 Cell Differentiation by Interleukin-1 Signaling," Immunity, vol. 30, no. 4, pp. 576-587, 2009.

[16] J. M. Damsker, A. M. Hansen, and R. R. Caspi, “Th1 and Th17 cells: adversaries and collaborators," Annals of the New York Academy of Sciences, vol. 1183, pp. 211-221, 2010.

[17] S. Kagami, H. L. Rizzo, J. J. Lee, Y. Koguchi, and A. Blauvelt, "Circulating Th17, Th22, and Th1 cells are increased in psoriasis," Journal of Investigative Dermatology, vol. 130, no. 5, pp. 1373-1383, 2010.

[18] E. Fitch, E. Harper, I. Skorcheva, S. E. Kurtz, and A. Blauvelt, "Pathophysiology of psoriasis: recent advances on IL-23 and TH17 cytokines," Current Rheumatology Reports, vol. 9, no. 6, pp. 461-467, 2007.

[19] Y. Iwakura and H. Ishigame, "The IL-23/IL-17 axis in inflammation," Journal of Clinical Investigation, vol. 116, no. 5, pp. 1218-1222, 2006.

[20] L. Naldi and D. Gambini, "The clinical spectrum of psoriasis," Clinics in Dermatology, vol. 25, no. 6, pp. 510-518, 2007.

[21] N. Balato, L. Di Costanzo, and A. Balato, "Differential diagnosis of psoriasis," Journal of Rheumatology, vol. 36, no. 83, pp. 24-25, 2009.

[22] P. Lisi, "Differential diagnosis of psoriasis," Reumatismo, vol. 59, pp. 56-60, 2007.

[23] P. Balasubramaniam and J. Berth-Jones, "Erythroderma: $90 \%$ skin failure," Hospital Medicine, vol. 65, no. 2, pp. 100-102, 2004.

[24] L. Puig-Sanz, "Psoriasis, a systemic disease?" Actas DermoSifiliograficas, vol. 98, no. 6, pp. 396-402, 2007.

[25] M. M. Farías, V. Serrano, and C. De La Cruz, "Psoriasis and obesity: a review and practical recommendations," Actas Dermo-Sifiliograficas, vol. 102, no. 7, pp. 505-509, 2011.

[26] A. B. Gottlieb and F. Dann, "Comorbidities in patients with psoriasis," American Journal of Medicine, vol. 122, no. 12, pp. 1150-e1, 2009.

[27] W. H. Boehncke, S. Boehncke, A. M. Tobin, and B. Kirby, "The "psoriatic march": a concept of how severe psoriasis may drive cardiovascular comorbidity," Experimental Dermatology, vol. 20, no. 4, pp. 303-307, 2011.

[28] A. C. Foulkes, D. J. C. Grindlay, C. E. M. Griffiths, and R. B. Warren, "What's new in psoriasis? an analysis of guidelines and systematic reviews published in 2009-2010," Clinical and Experimental Dermatology, vol. 36, no. 6, pp. 585-588, 2011.

[29] A. M. Tobin, D. J. Veale, O. FitzGerald et al., "Cardiovascular disease and risk factors in patients with psoriasis and psoriatic 
arthritis," Journal of Rheumatology, vol. 37, no. 7, pp. 13861394, 2010.

[30] S. Prey, C. Paul, V. Bronsard et al., "Cardiovascular risk factors in patients with plaque psoriasis: a systematic review of epidemiological studies," Journal of the European Academy of Dermatology and Venereology, vol. 24, supplement 2, pp. 2330, 2010.

[31] N. Balato, L. Di Costanzo, A. Balato, C. Patruno, M. Scalvenzi, and F. Ayala, "Psoriasis and melanocytic naevi: does the first confer a protective role against melanocyte progression to naevi?" British Journal of Dermatology, vol. 164, no. 6, pp. 1262-1270, 2011.

[32] B. B. Yeap, S. A. Paul Chubb, K. A. McCaul et al., "Associations of igfl and igfbps 1 and 3 with all-cause and cardiovascular mortality in older men: the health in men study," European Journal of Endocrinology, vol. 164, no. 5, pp. 715-723, 2011.

[33] A. Damasiewicz-Bodzek, B. Kos-Kudła, and B. SuwałaJurczyk, "Somatotrophin axis hormones in patients affected with psoriasis," Neuroendocrinology Letters, vol. 26, no. 6, pp. 724-728, 2005.

[34] S. Savastano, N. Balato, F. Gaudiello et al., "Insulin-like growth factor-1, psoriasis, and inflammation: a ménage à trois?" European Journal of Inflammation, vol. 9, no. 3, pp. 277-283, 2011.

[35] R. V. Patel, L. N. Clark, M. Lebwohl, and J. M. Weinberg, "Treatments for psoriasis and the risk of malignancy," Journal of the American Academy of Dermatology, vol. 60, no. 6, pp. 1001-1017, 2009.

[36] S. L. Brown, M. H. Greene, S. K. Gershon, E. T. Edwards, and M. M. Braun, "Tumor necrosis factor antagonist therapy and lymphoma development: twenty-six cases reported to the Food and Drug Administration," Arthritis and Rheumatism, vol. 46, no. 12, pp. 3151-3158, 2002.

[37] A. C. Esser, A. Abril, S. Fayne, and J. A. Doyle, "Acute development of multiple keratoacanthomas and squamous cell carcinomas after treatment with infliximab," Journal of the American Academy of Dermatology, vol. 50, no. 5, supplement, pp. S75-S77, 2004.

[38] N. M. Patkar, G. G. Teng, J. R. Curtis, and K. G. Saag, "Association of infections and tuberculosis with antitumor necrosis factor alpha therapy," Current Opinion in Rheumatology, vol. 20, no. 3, pp. 320-326, 2008.

[39] C. G. Grijalva, L. Chen, E. Delzell et al., "Initiation of tumor necrosis factor- $\alpha$ antagonists and the risk of hospitalization for infection in patients with autoimmune diseases," Journal of the American Medical Association, vol. 306, no. 21, pp. 2331-2339, 2011.

[40] E. D. Dommasch, K. Abuabara, D. B. Shin, J. Nguyen, A. B. Troxel, and J. M. Gelfand, "The risk of infection and malignancy with tumor necrosis factor antagonists in adults with psoriatic disease: a systematic review and meta-analysis of randomized controlled trials," Journal of the American Academy of Dermatology, vol. 64, no. 6, pp. 1035-1050, 2011.

[41] V. Bordignon, S. Bultrini, G. Prignano et al., "High prevalence of latent tuberculosis infection in autoimmune disorders such as psoriasis and in chronic respiratory diseases, including lung cancer," Journal of Biological Regulators and Homeostatic Agents, vol. 25, no. 2, pp. 213-220, 2011.

[42] I. D. Bassukas, M. Kosmidou, G. Gaitanis, G. Tsiouri, and A. E. Tsianos, "Patients with psoriasis are more likely to be treated for latent tuberculosis infection prior to biologics than patients with inflammatory bowel disease," Acta DermatoVenereologica, vol. 91, no. 4, pp. 444-446, 2011.
[43] M. Sánchez-Regaña, E. Dilmé, L. Puig et al., "Adverse reactions during biological therapy for psoriasis: results of a survey of the Spanish Psoriasis Group," Actas Dermo-Sifiliograficas, vol. 101, no. 2, pp. 156-163, 2010.

[44] D. Salmon-Ceron, F. Tubach, O. Lortholary et al., "Drugspecific risk of non-tuberculosis opportunistic infections in patients receiving anti-TNF therapy reported to the 3-year prospective French RATIO registry," Annals of the Rheumatic Diseases, vol. 70, pp. 616-623, 2011.

[45] F. Tubach, D. Salmon, P. Ravaud et al., "Risk of tuberculosis is higher with anti-tumor necrosis factor monoclonal antibody therapy than with soluble tumor necrosis factor receptor therapy: the three-year prospective French research axed on tolerance of biotherapies registry," Arthritis and Rheumatism, vol. 60, no. 7, pp. 1884-1894, 2009.

[46] J. E. Fonseca, H. Canhão, C. Silva et al., "Tuberculosis in rheumatic patients treated with tumour necrosis factor alpha antagonists: the Portuguese experience," Acta Reumatológica Portuguesa, vol. 31, no. 3, pp. 247-253, 2006.

[47] A. I. Sánchez-Moya and E. Dauden, "Incidence of tuberculosis infection in psoriatic patients on anti-tnf therapy: report of a case series with 144 patients," Journal of the European Academy of Dermatology and Venereology, vol. 25, no. 6, pp. 730-733, 2011.

[48] I. Solovic, M. Sester, J. J. Gomez-Reino et al., "The risk of tuberculosis related to tumour necrosis factor antagonist therapies: a TBNET consensus statement," European Respiratory Journal, vol. 36, no. 5, pp. 1185-1206, 2010.

[49] J. L. Perez, H. Kupper, and G. Spencer-Green, "Impact of screening for latent TB prior to initiation of anti-TNF therapy in North America and Europe," Annals of the Rheumatic Diseases, vol. 64, pp. 86-90, 2005.

[50] L. Carmona, J. J. Gómez-Reino, V. Rodríguez-Valverde et al., "Effectiveness of recommendations to prevent reactivation of latent tuberculosis infection in patients treated with tumor necrosis factor antagonists," Arthritis and Rheumatism, vol. 52, no. 6, pp. 1766-1772, 2005.

[51] American Thoracic Society (ATS) and the Centers for Disease Control and Prevention (CDC), "Targeted tuberculin skin testing and treatment of latent tuberculosis infection," American Journal of Respiratory and Critical Care Medicine, vol. 161, no. 4, pp. 5221-5247, 2000.

[52] Centers for Disease Control and Prevention (CDC), "Tuberculosis associated with blocking agents against tumor necrosis factor-alpha-California, 2002-2003," Morbidity and Mortality Weekly Report, vol. 53, no. 30, pp. 683-686, 2004.

[53] D. Menzies, "Interpretation of repeated tuberculin tests: boosting, conversion, and reversion," American Journal of Respiratory and Critical Care Medicine, vol. 159, no. 1, pp. 1521, 1999.

[54] L. P. Ormerod, H. J. Milburn, S. Gillespie, J. Ledingham, and D. Rampton, "BTS recommendations for assessing risk and for managing Mycobacterium tuberculosis infection and disease in patients due to start anti-TNF- $\alpha$ treatment," Thorax, vol. 60, no. 10, pp. 800-805, 2005.

[55] A. Lalvani and K. A. Millington, "Screening for tuberculosis infection prior to initiation of anti-TNF therapy," Autoimmunity Reviews, vol. 8, no. 2, pp. 147-152, 2008.

[56] S. Moyo, F. Isaacs, S. Gelderbloem et al., "Tuberculin skin test and QuantiFERON assay in young children investigated for tuberculosis in South Africa," International Journal of Tuberculosis and Lung Disease, vol. 15, no. 9, pp. 1176-1181, 2011. 
[57] J. H. Chung, C. H. Han, C. J. Kim, and S. M. Lee, "Clinical utility of QuantiFERON-TB GOLD In-Tube and tuberculin skin test in patients with tuberculous pleural effusions," Diagnostic Microbiology and Infectious Disease, vol. 71, no. 3, pp. 263-266, 2011.

[58] R. Diel, R. Loaddenkemper, and A. Nienhaus, "EvidenceBased comparison of commercial Interferon- $\gamma$ Release assays for detecting active TB a metaanalysis," Chest, vol. 137, no. 4, pp. 952-968, 2010.

[59] R. N. van Zyl-Smit, A. Zwerling, K. Dheda, and M. Pai, "Within-subject variability of interferon-g assay results for tuberculosis and boosting effect of tuberculin skin testing: a systematic review," PLoS ONE, vol. 4, no. 12, Article ID e8517, 2009.

[60] M. Bocchino, B. Bellofiore, A. Matarese, D. Galati, and A. Sanduzzi, "IFN- $\gamma$ release assays in tuberculosis management in selected high-risk populations," Expert Review of Molecular Diagnostics, vol. 9, no. 2, pp. 165-177, 2009.

[61] G. Tsiouri, G. Gaitanis, D. Kiorpelidou et al., "Tuberculin skin test overestimates tuberculosis hypersensitivity in adult patients with psoriasis," Dermatology, vol. 219, no. 2, pp. 119$125,2009$.

[62] J. D. Bos, M. A. De Rie, M. B. M. Teunissen, and G. Piskin, "Psoriasis: dysregulation of innate immunity," British Journal of Dermatology, vol. 152, no. 6, pp. 1098-1107, 2005.

[63] S. Katsenos, M. Nikolopoulou, G. Tsiouri, I. D. Bassukas, and S. H. Constantopoulos, "The challenging evaluation of patients with severe psoriasis for latent tuberculosis: an important indication for IGRA," Open Respiratory Medicine Journal, vol. 5, no. 1, pp. 59-60, 2011.

[64] Y. Z. Chiang, K. Panting, B. Dever, and R. A. G. Parslew, "Clinical applicability of T-cell interferon- $\alpha$ release assay for tumour necrosis factor- $\alpha$ inhibitor therapy in severe psoriasis," Clinical and Experimental Dermatology, vol. 36, no. 1, pp. 3941, 2011.

[65] E. Laffitte, J. P. Janssens, P. Roux-Lombard et al., “Tuberculosis screening in patients with psoriasis before antitumour necrosis factor therapy: comparison of an interferon- $\gamma$ release assay vs. tuberculin skin test," British Journal of Dermatology, vol. 161, no. 4, pp. 797-800, 2009.

[66] H.-Y. Chiu, P.-R. Hsueh, and T.-F. Tsai, "Clinical experience of QuantiFERON-TB Gold testing in patients with psoriasis treated with tumour necrosis factor blockers in Taiwan," British Journal of Dermatology, vol. 164, no. 3, pp. 553-559, 2011.

[67] R. J. Bowerman, "Tuberculin skin testing in BCG-vaccinated populations of adults and children at high risk for tuberculosis in Taiwan," International Journal of Tuberculosis and Lung Disease, vol. 8, no. 10, pp. 1228-1233, 2004.

[68] E. de Andrade Lima, M. de Andrade Lima, V. M.B. de Lorena, Y. de Miranda Gomes, O. Lupi, and G. Benard, "Evaluation of an IFN-gamma assay in the diagnosis of latent tuberculosis in patients with psoriasis in a highly endemic setting," Acta Dermato-Venereologica, vol. 91, no. 6, pp. 694-697, 2011.

[69] L. C. R. Silva, G. G. Silveira, M. Arnone et al., "Decrease in Mycobacterium tuberculosis specific immune responses in patients with untreated psoriasis living in a tuberculosis endemic area," Archives of Dermatological Research, vol. 302, no. 4, pp. 255-262, 2010.

[70] H. H. Oon, W. S. Chong, and C. F. Liew, "Indeterminate results on the interferon- $\gamma$ release assay for tuberculosis screening should not be ignored," British Journal of Dermatology, vol. 159, no. 4, pp. 992-993, 2008.
[71] M. Bocchino, A. Matarese, B. Bellofiore et al., "Performance of two commercial blood IFN- $\gamma$ release assays for the detection of Mycobacterium tuberculosis infection in patient candidates for anti-TNF- $\alpha$ treatment," European Journal of Clinical Microbiology and Infectious Diseases, vol. 27, no. 10, pp. 907-913, 2008.

[72] N. Balato, F. Ayala, F. Gaudiello et al., "Comparison of tuberculin skin test and interferon- $\gamma$ assays in patients with moderate to severe psoriasis who are candidates for antitumour necrosis factor- $\alpha$ therapy," British Journal of Dermatology, vol. 158, no. 4, pp. 847-849, 2008.

[73] B. Bellofiore, A. Matarese, N. Balato et al., "Prevention of tuberculosis in patients taking tumor necrosis factor- $\alpha$ blockers," Journal of Rheumatology, vol. 36, no. 83, pp. 76-77, 2009.

[74] M. M. Haddican and J. Y. M. Koo, "Is tuberculin skin testing reliable during anti-tumor necrosis factor-alpha therapy? A case report and review of the literature," Journal of the American Academy of Dermatology, vol. 65, no. 1, pp. 1951197, 2009.

[75] F. Bartalesi, S. Vicidomini, D. Goletti et al., "QuantiFERONTB Gold and the TST are both useful for latent tuberculosis infection screening in autoimmune diseases," European Respiratory Journal, vol. 33, no. 3, pp. 586-593, 2009.

[76] S. Garcovich, A. Ruggeri, and M. D’Agostino, "Clinical applicability ofQuantiferon-TB-Gold testing in psoriasis patients during long-term anti-TNF-alpha treatment: a prospective, observational study," Journal of the European Academy of Dermatology and Venereology. In press.

[77] G. Matulis, P. Jüni, P. M. Villiger, and S. D. Gadola, "Detection of latent tuberculosis in immunosuppressed patients with autoimmune diseases: performance of a Mycobacterium tuberculosis antigen-specific interferon $\gamma$ assay," Annals of the Rheumatic Diseases, vol. 67, no. 1, pp. 84-90, 2008.

[78] H. Hamdi, X. Mariette, V. Godot et al., "Inhibition of antituberculosis T-lymphocyte function with tumour necrosis factor antagonists," Arthritis Research and Therapy, vol. 8, no. 4, article no. R114, 2006.

[79] D. Y. Chen, G. H. Shen, T. Y. Hsieh, C. W. Hsieh, and J. L. Lan, "Effectiveness of the combination of a wholeblood interferon-gamma assay and the tuberculin skin test in detecting latent tuberculosis infection in rheumatoid arthritis patients receiving adalimumab therapy," Arthritis Care and Research, vol. 59, no. 6, pp. 800-806, 2008.

[80] A. I. Sanchez-Moya and E. Dauden, "Diagnosing latent tuberculosis infection in patients with psoriasis under antitumour necrosis factor- $\alpha$ treatment: every new solution breeds new doubts," British Journal of Dermatology, vol. 164, no. 1, pp. 208-209, 2011.

[81] G. H. Mazurek, J. Jereb, P. Lobue, M. F. Iademarco, B. Metchock, and A. Vernon, "Guidelines for using the QuantiFERON-TB Gold test for detecting Mycobacterium tuberculosis infection, United States," Morbidity and Mortality Weekly Report, vol. 54, no. 15, pp. 49-55, 2005.

[82] G. H. Mazurek, J. Jereb, A. Vernon, P. LoBue, S. Goldberg, and K. Castros, "Updated guidelines for using interferon gamma release assays to detect Mycobacterium tuberculosis infectionUnited States, 2010," Morbidity and Mortality Weekly Report, vol. 59, no. 5, pp. 1-25, 2010.

[83] National Collaborating Centre for Chronic Conditions, Tuberculosis: Clinical Diagnosis and Management of Tuberculosis, and Measures for Its Prevention and Control, Royal College of Physicians, London, UK, 2006.

[84] HPA Tuberculosis Programme Board (Health Protection Agency), "Health Protection Agency position statement: on 
the use of interferon- $\gamma$ release assay (IGRA) tests for tuberculosis (TB): draft for consultation," October 2007.

[85] Canadian Tuberculosis Committee, "Updated recommendations on interferon- $\gamma$ release assay for latent tuberculosis infection: an advisory committee statement (ACS)," Canada Communicable Disease Report, vol. 34, no. ACS-6, pp. 1-13, 2008.

[86] Documento sull'utilizzo dei nuovi test immunologici per la diagnosi di infezione tubercolare latente, Elaborato dei Gruppi di Studio Infezioni e Tubercolosi dell'Associazione Italiana Pneumologi Ospedalieri (AIPO) e della Società Italiana di Medicina Respiratoria (SIMeR), http://www.simernet.it/.

[87] J. E. Fonseca, H. Lucas, H. Canhão et al., "Recommendations for the diagnosis and treatment of latent and active tuberculosis in inflammatory joint diseases candidates for therapy with tumor necrosis factor alpha inhibitors- March 2008 update," Revista Portuguesa de Pneumologia, vol. 14, no. 2, pp. 271-283, 2008.

[88] S. D. Doherty, A. Van Voorhees, M. G. Lebwohl, N. J. Korman, M. S. Young, and S. Hsu, "National Psoriasis Foundation consensus statement on screening for latent tuberculosis infection in patients with psoriasis treated with systemic and biologic agents," Journal of the American Academy of Dermatology, vol. 59, no. 2, pp. 209-217, 2008.

[89] O. Elkayam, A. Balbir-Gurman, M. Lidgi, G. Rahav, and D. Weiler-Ravel, "Guidelines of the Israeli Association of Rheumatology for the prevention of tuberculosis in patients treated with Tnf-Á blockers," Harefuah, vol. 146, no. 3, pp. 235-237, 2007.

[90] X. Mariette and D. Salmon, "French guidelines for diagnosis and treating latent and active tuberculosis in patients with RA treated with TNF blockers," Annals of the Rheumatic Diseases, vol. 62, no. 8, p. 791, 2003.

[91] P. M. Kavanagh, J. J. Glimartin, J. O’Donnell, and D. O'Flanagan, “Tumour Necrosis Factor-a and tuberculosis: guidance from the National TB Advisory Committee," Irish Medical Journal, vol. 101, no. 1, pp. 6-7, 2008.

[92] C. Beglinger, J. Dudler, C. Mottet et al., "Screening for tuberculosis infection before initiation of anti-TNF- $\alpha$ therapy," Swiss Medical Weekly, vol. 137, no. 43-44, pp. 621-622, 2007.

[93] R. Diel, B. Hauer, R. Loddenkemper, B. Manger, and K. Krüger, "Recommendations for tuberculosis screening before initiation of TNF - inhibitor treatment in rheumatic diseases," Pneumologie, vol. 63, no. 6, pp. 329-334, 2009.

[94] "Diagnostic Standards and Classification of Tuberculosis in Adults and Children. This official statement of the American Thoracic Society and the Centers for Disease Control and Prevention was adopted by the ATS Board of Directors, July 1999. This statement was endorsed by the Council of the Infectious Disease Society of America, September 1999," American Journal of Respiratory and Critical Care Medicine, vol. 161, no. 4, pp. 1376-1395, 2000. 


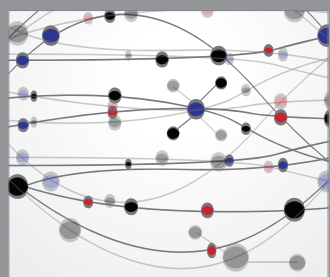

The Scientific World Journal
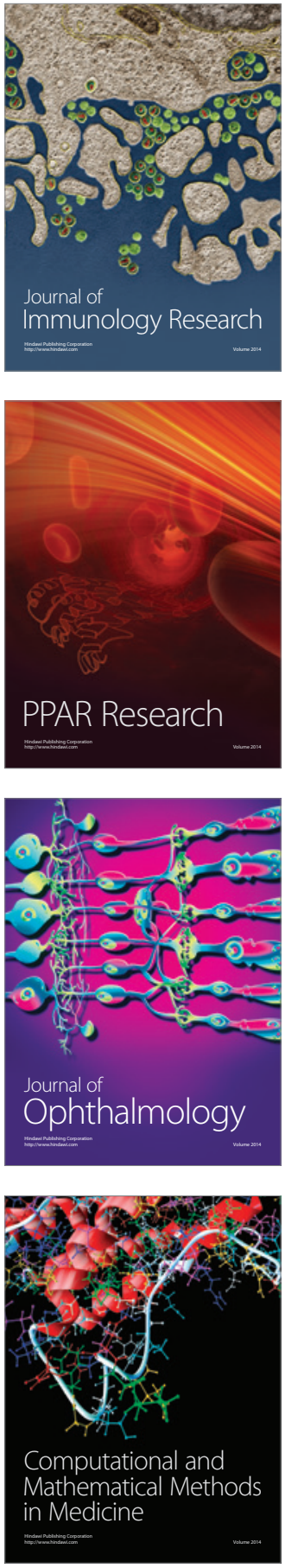

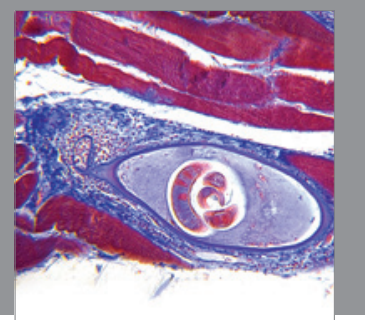

Gastroenterology

Research and Practice
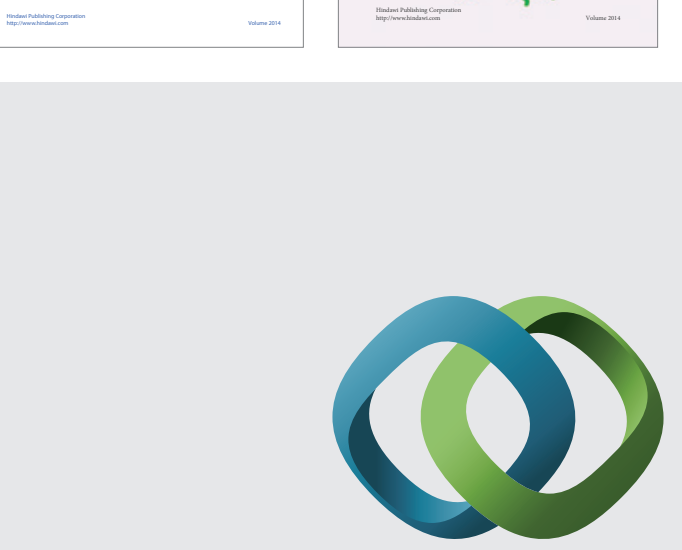

\section{Hindawi}

Submit your manuscripts at

http://www.hindawi.com
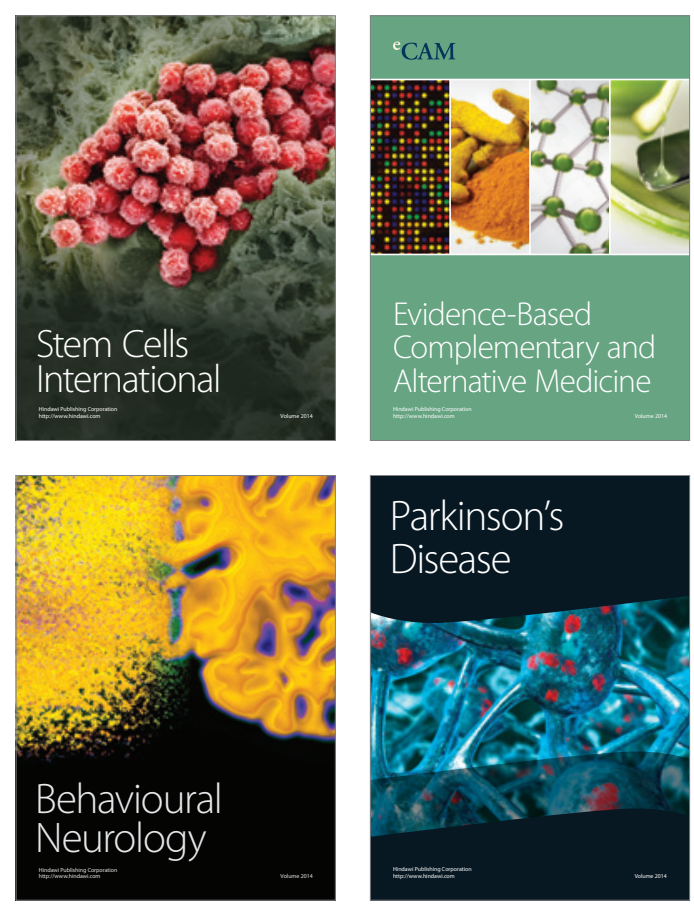

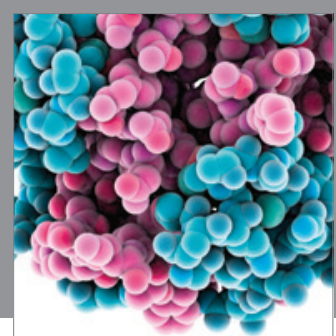

Journal of
Diabetes Research

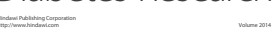

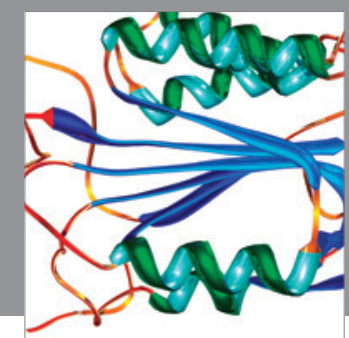

Disease Markers
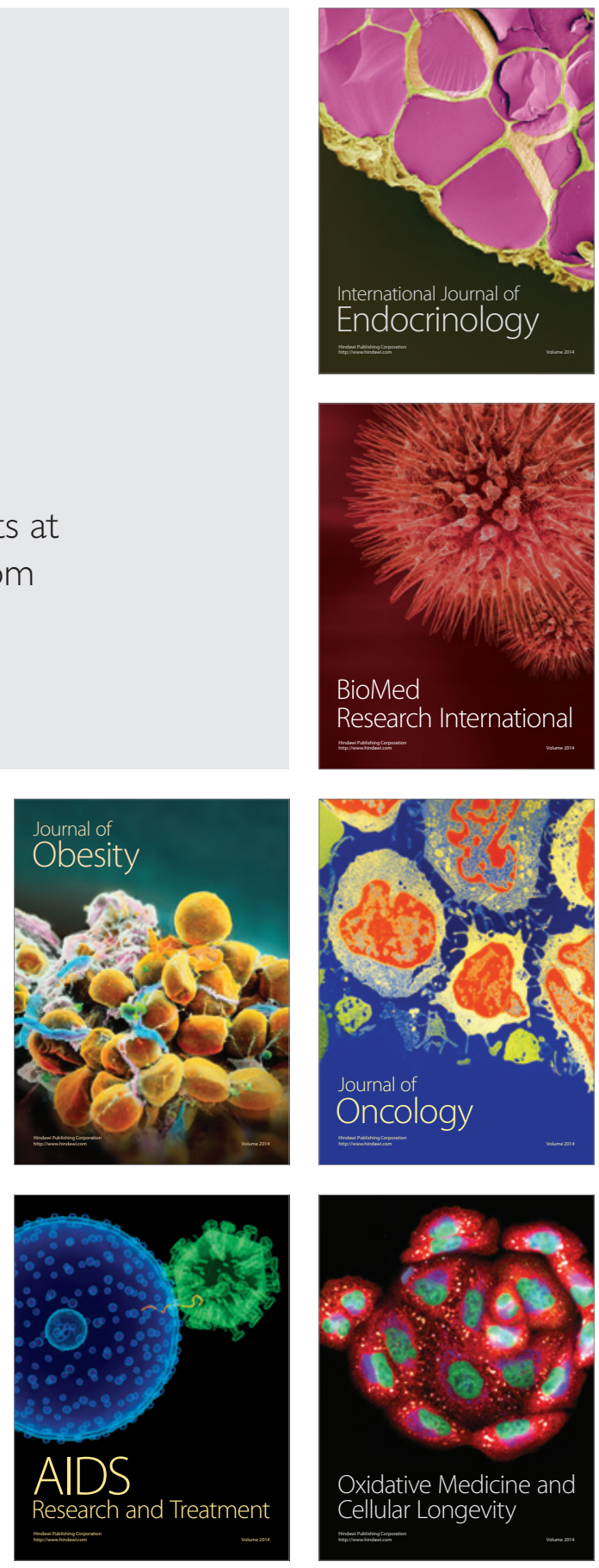